KOŚCIÓŁ I PRAWO 8(21) 2019, nr 1, s. 231-247

DOI: http://dx.doi.org/10.18290/kip.2019.8.1-15

\author{
Mykhailo Klapkiv
}

\title{
DIE AUSWIRKUNGEN DES KONKORDATS ZWISCHEN DEM APOSTOLISCHEN STUHL UND POLEN VON 1925 AUF DIE EPARCHIE STANISLAVIV
}

Die Zwischenkriegszeit in Galizien ist durch zahlreiche politisch-gesellschaftliche Prozesse gekennzeichnet. Am Ende des Ersten Weltkrieges, während des Zerfalls der österreichisch-ungarischen Monarchie und der Bildung neuer nationaler Staaten in Mittelosteuropa, verschärfte sich die ethno-politische Situation in Galizien beträchtlich. Die Bischöfe der Griechisch-Katholischen Kirche unterstützten die Proklamation der Westukrainischen Volksrepublik und die staatsbildenden Prozesse in der Region. Sie verurteilten auch die antiukrainischen Orientierungen von Seiten der Regierung des erneuerten polnischen Staates. In der durch das Dekret des Kaisers Franz Josef I und der Bulle vom Papst Leo XIII 1885 errichteten griechisch-katholischen Eparchie Stanislau wurde ein Programm der ukrainisch-polnischen Verständigung vorgebracht, welches während einer zunehmenden Spannung zwischen den beiden Völkern am Anfang der 1920er Jahre zu stark klerikalisiert und dementsprechend auch nicht realisiert wurde. Trotz aller Aktivitäten der diplomatischen Missionen der Westukrainischen Volksrepublik und trotz der Proteste und des Appells der Öffentlichkeit fasste der Gesandtenrat der Entente am 15. März 1923 endgültig den Beschluss, Ostgalizien unter polnisches Protektorat zu stellen ${ }^{1}$.

ReV. MYKhailo KlaPkiv, J.C.L. - Ph.D. student, Department of Law of the Eastern Catholic Churches, Institute of Canon Law, Faculty of Law, Canon Law and Administration at the John Paul II Catholic University of Lublin; Al. Racławickie 14, 20-950 Lublin, Poland; e-mail: mykhailo.klapkiv@ukr.net; https://orcid.org/0000-0001-5276-5265

1 Vgl. Svoboda [Organ der Ukrainischen Nationaldemokratischen Partei], Nr. 260 Lviv 1922. 
Solch eine Konstellation hatte gewiss zu Auswirkungen in Ostgalizien, bzw. in den drei griechisch-katholischen (Erz-) Eparchien geführt - der Erzeparchie Lviv, Eparchie Peremyšl und Eparchie von Stanislau. Unter vielen Veränderungen war eines von enormer Bedeutung - die Verlautbarung zwischen dem Apostolischen Stuhl und dem polnischen Staat - Konkordat von 1925.

Der Begriff des Konkordats war in der Zwischenkriegszeit sehr oft zu hören. So wurden zahlreiche Verlautbarungen zwischen der Katholischen Kirche und den europäischen Staaten geschlossen (Bayern 1922, Preußen, Rumänien, Lettland, Frankreich, Portugal, Italien 1929). Dies hat sich besonders im Sinne der östlichen Kirchenpolitik des Papstes Benedikt XV und des Pius XI auf dem Konkordat mit Polen von 1925 erwiesen.

\section{VORBEDINGUNGEN ZUR AUSARBEITUNG VON KONKORDAT}

Seit dem März 1923 gehörten ostgalizische Gebiete zur zweiten polnischen Republik. Dies bedeutete vor allem eine Umstellung für die Griechisch-Katholische Kirche, die bisher fast 150 Jahre lang ihre Beziehungen mit dem anderen zentraleuropäischen Staat geführt hat, und zwar mit der kaiserlichen und königlichen Monarchie (k. u. k. Monarchie) - Österreich-Ungarn.

Möchte man die wichtigsten Ursachen und Vorbedingungen benennen, die in der Entstehung des Konkordats seitens der Ukrainischen Griechisch-Katholischen Kirche (UGKK) wahrgenommen wurden, erweist sich als wichtig, auf drei unterschiedlichen Aspekten einen Akzent zu setzten.

\subsection{Rechtsunsicherheit}

Solch eine geopolitische Änderung verursachte einen im gewissen Sinne rechtlichen Nihilismus in Bezug auf das Staatskirchenrecht, was sich im Sinne einer selektiven Gerechtigkeit erwies. So galten für die kirchlich-staatliche Beziehungen die Paragraphen der polnischen Verfassung von 1921. Andererseits gültig war für die polnische Regierung auch der Gesetzeskorpus aus der k. u. k. Monarchie von 1874, deren Verlautbarungen nach Beliebigkeit selektiv angewendet wurde. So kam es immer wieder zu Missverständnissen bei der Beauftragung oder Umsetzung von Pfarrern. Dies 
Die Auswirkungen des Konkordats zwischen dem Apostolischen Stuhl 233

führe dazu, dass zahlreiche Pfarreien auch im Bistum Stanislau für lange Zeit vakant blieben [Hentosh 2016b, 21-25]. Die griechisch-katholische Kirche hat auf diese Lage stets die Öffentlichkeit und Regierungsmächte aufmerksam gemacht. So wandte sich am 20. Juni 1924 die St. Paul Priestergesellschaft der Erzeparchie Lviv an die Liga der Nationen mit der Beschwerde, wonach viele unierte Priester aufgrund ihrer öffentlichen Position von pastoralen Verantwortungsaufgaben ausgenommen wurden.

Sehr streng hat der polnischen Regierung, bspw. dem Wojewoden von Lviv daran gelegen, dass die Griechisch-Katholischen die Taufzeugnisse in Latein abfassen und unbedingt die Spalte „Staat“ mit „Polen“ ausfühlen [ibidem]. Dies wurde streng kontrolliert, sodass die Taufbücher mit Gewalt aus der St. Georgs Kathedrale am 10. Januar 1922 seitens der Vertretung der Ortpolizei entnommen wurden. An dem Beispiel soll verdeutlicht werden, dass die Rechtsverhältnisse zwischen den beiden Subjekten - Kirche und Staat - neu geklärt werden sollten. Dies betraf selbstverständlich auch die UGKK.

\subsection{Finanzielle und wirtschaftliche Schwierigkeiten}

Ein dringendes Problem, das eine schnelle Lösung erforderte, war das Thema der materialen und finanziellen Lage. Der Erzbischof und Metropolit von Lviv Andrei Šeptytsky (1865-1944) übernahm seine Metropolie in materieller Notlage, die durch Kriese und Zerstörung der Nachkriegszeit sowie eines starken Rückgangs der staatlichen Finanzhilfe ausgezeichnet und enorm geschwächt war.

Die katholische Kirche in der österreich-ungarischen Zeit profitierte von großer staatlicher Unterstützung, da jeder Geistliche vom Staat Geld erhielt und auch die Witwen und Waisen von verstorbenen Priestern eine Rente bekamen. Darüber hinaus verfügte die Erzeparchie Lviv mit den anderen Eparchien über ein großes Immobilienvermögen und Landbesitz, die von Diözesanbischöfen und der Kapitel frei verwaltet wurden. Nun hat sich nach dem Ersten Weltkrieg die finanzielle der UGKK im generellen und des griechisch-katholischen Klerus im speziellen rasant verschlechtert. Erhielt ein griechisch-katholischer Bischof oder Erzbischof, der in Österreich-Ungarn eine jährliche staatliche Beihilfe von 24.000 Kronen pro Jahr, wurde ihm zwischen 1922 und 19231.007 polnische Mark pro Monat anerkannt, die durch die galoppierende Inflation abgewertet wur- 
den [ibidem]. Die einzige Haupteinnahmenquelle war für die unierte Kirche das Immobilienvermögen, was in der zeitlichen Periode von großer Bedeutung war.

\subsection{Moral-ethische Frage}

Rechtsunsicherheit sowie finanzielle und wirtschaftliche Schwierigkeiten wurden durch die komplexe moralische und ethische Situation ergänzt, in der griechisch-katholische Klerus auftrat. Die Unterstützung der ukrainischen Gemeinschaft und eines großen Teils des griechisch-katholischen Klerus im ukrainischen Befreiungskampf galt als anti-staatliche Aktivität. Alle Aktionen der ukrainischen Partei-, Bürger- und Kirchenkreise, die bis zum 14. März 1923 gegen Maßnahmen der polnischen Regierung gekämpft hatten, wie der ukrainische Boykott der Volkszählung von 1921 und die Wahlen zum Sejm von 1922, wurden als Bedrohung auf die Unabhängigkeit Polens und seine territoriale Integrität eingestuft. Die ukrainischen Widerstandshandlungen wollten ihrerseits zur Souveränität des ukrainischen Staates beitragen.

Metropolit Šeptytsky glaubte seinerseits auch, dass seine aktive Tätigkeit auf internationaler Ebene für die Unterstützung des Westukrainischen Volksrepublik nicht als Versuch der Unabhängigkeit Polens eingestuft werden darf. Dies war die Meinung auch vieler ukrainischer Kleriker, die zwar eine öffentlich-politische Stellung zur Unterstützung der ukrainischen Unabhängigkeit einnahmen, andererseits wollten keine separatistischen Tendenzen zeigen.

Für die polnische Regierung und die lokale Verwaltung wurden der griechisch-katholische Klerus und sein Oberhaupt a priori anti-staatlicher Aktivität in anti-staatlichen Handlungen zumindest verdächtigt. Vertreter der örtlichen Verwaltung und der Polizei verfolgten das Verhalten der griechisch-katholischen Geistlichen aufmerksam und bewerteten die Loyalität des griechisch-katholischen Geistes und des Episkopats.

Im November 1922 verfassten die Hierarchen der UGKK zusammen mit den bekannten gesellschaftlichen Politikern ein Schreiben an die ganze Kulturwelt anlässlich des vierten Jahrestages der polnischen Besatzung. Die Verfasser verurteilten die repressive Politik der polnischen Obrigkeit und wiesen darauf hin, dass vor den Augen des ukrainischen 
Volkes eine schreckliche Vision erscheint: Der Entzug der Menschenrechte und die Entnationalisierung im eigenen Heimatland. Trotz aller Aktivitäten der diplomatischen Missionen der Westukrainischen Volksrepublik und trotz der Proteste und des Appells der Öffentlichkeit fasste der Gesandtenrat der Entente am 15. März 1923 endgültig den Beschluss, Ostgalizien unter polnisches Protektorat zu stellen².

Um über die Lage in Galizien im Bilde zu sein, achteten die Bezirks- und Wojewodschaftsverwaltungen auf die Stimmungen der Ukrainer, unter ihnen auch auf jene des griechisch-katholischen Klerus und der Bischöfe. Wie die Meldungen aus verschiedenen Orten einhellig bezeugen, war das Verhalten der Bevölkerung und des Klerus gegenüber den polnischen Machthabern in fast allen Bezirken meistens feindlich. So waren beispielsweise in der Wojewodschaft Stanislau von 588 Priestern nur 154 der polnischen Macht gegenüber loyal, 108 verhielten sich politisch passiv und beschäftigten sich ausschließlich mit kirchlichen Angelegenheiten, 200 nahmen gegenüber den polnischen Machthabern eine unfreundliche Haltung ein. Der Wojewode war der Meinung, dass diese Sachlage darauf zurückzuführen war, dass auch das Verhalten der griechisch-katholischen Hierarchen gegenüber der Macht nicht völlig loyal war. So war die Rate der mit der polnischen Macht unzufriedenen Priester in der Eparchie Stanislau am niedrigsten, am größten dagegen in der Erzeparchie Lviv. Der Wojewode erklärte das damit, dass der Stanislauer Bischof Hryhorij Chomyšyn (18671945) mehr Aufmerksamkeit dem kirchlichen Leben als der Politik schenkte. Demgegenüber schlossen sich Metropolit Andrei und Bischof Josafat Kocylovskyj (1876-1947) von Peremyšl der für den polnischen Staat schädlichen Bewegung zur Wiedererweckung des ukrainischen Geistes und der Erneuerung der Westukrainischen Volksrepublik an [Jehreshij 2003, 77].

Solch eine Lage hat die Brisanz und die Wichtigkeit der diplomatischen und bürokratischen Bemühungen zwischen Kirche und Staat für notwendig gemacht. Diese komplexen Fragen sollte das im Februar 1925 unterzeichnete Konkordat zwischen dem polnischen Staat und dem Apostolischen Stuhl lösen.

${ }^{2}$ Vgl. Svoboda [Organ der Ukrainischen Nationaldemokratischen Partei], Nr. 260 Lviv 1922. 


\section{EINFLUSS DER UGKK AUF DIE KONKORDATSENTWÜRFE}

Die Vorbereitungen und Entwürfe des Konkordats mit Polen haben viel Zeit in Anspruch genommen. Die zweite polnische Republik war in der Tat multinational sowie multikonfessionell und die staatliche Politik in religiösen und konfessionellen Angelegenheiten diente häufig als Instrument der nationalen Politik.

Als der Hl. Stuhl 1919 Verhandlungen mit Vertretern der polnischen Regierung über das Konkordat aufnahm, wurde er von einigen polnischen Bischöfen (Lviver armenischer Erzbischof Joseph Teodorovich, Bischof von Krakau Adam Sapieha, Erzbischof von Gniezno und Posen Edmund Dalbor) angegriffen. Diese Gruppe einflussreicher Bischöfe wollte mit der Regierung selbst verhandeln, was weder die römische Kurie noch die polnische Regierung wollte [Hentosh 2016a, 115-28].

Weder die Regierung noch der Heilige Stuhl haben Angaben zum Inhalt und Prozess der Verhandlungen über den ersten Entwurf gemacht [Mróz 2004, 154]. Dies erschwerte die Objektivität und Transparenz der Vorbereitungen im Augen des katholischen Episkopats Polens, und zwar verschiedener Riten. Nichtsdestotrotz bemühten sich die kirchlichen Oberen, selbst mit dem Heiligen Stuhl und der polnischen Regierung Kontakt herzustellen und somit ihren Einfluss auf die Erarbeitung von Schemata für das wichtige Dokument möglich zu machen. Die Griechisch-Katholische Kirche hat in diesem Prozess Metropolit Šeptytsky vorgestellt, der im Namen seiner Bischöfe und Gläubigen aus der Suffraganbistümern Stanislau und Peremyšl gesprochen hat.

Auch der Stanislauer Bischof Hryhorij Chomyšyn (1867-1945) verfolgte das Ziel, die UGKK vor möglichen Verfolgungen zu schützen und seine Gläubigen vor orthodoxen Einflüssen aus dem Osten zu bewahren. Darum hielt er es für notwendig, mit der polnischen Regierung eine angemessene Vereinbarung zu schließen, welche den Status der griechisch-katholischen Kirche und deren Rechte und Pflichten im polnischen Staat bestimmen würde. Diese Idee teilte auch der römisch Apostolische Stuhl, der im Sinne des I. Vatikanischen Konzils sich auf das Codex Iuris Canonici von 1917 berufen hat, das die zentrealistische Positionen des Heiligen Stuhls im Sinne gemäßigten Ultramontanismus vorgestellt hat [Wolf 2010]. An der Abfassung dieser Vereinbarung beteiligte sich der Bischof am aktivsten. Die Vorschläge zu der Vereinbarung wurden auf einer erweiterten 
Die Auswirkungen des Konkordats zwischen dem Apostolischen Stuhl 237

Beratung beim Metropoliten am 19. März 1924 geprüft und gebilligt. An dieser Zusammenkunft nahmen auch die Vertreter der weltlichen Elite teil [Perewesij 1998, 20].

Betrachtet man die wichtigsten Aspekte der UGKK in der Vorbereitungsphase des Konkordats, ist es notwendig auf einige Punkte besondere Aufmerksamkeit zu wenden.

\subsection{Agrarreform}

In den Ländern, die zum ehemaligen österreichisch-ungarischen Reich gehörten, besaß die griechisch-katholische Kirche mehr Landbesitz als die Katholische Kirche in den Gebieten, die in dem russischen und preußischen Reich gehörten. Für den UGKK und seine Geistlichen war der Landbesitz von besonderer Bedeutung, da das Einkommen aus aufgerichteten Böden traditionell und gemäß österreichisch-ungarischem Recht einen erheblichen Anteil am Gesamteinkommen des griechisch-katholischen Pfarrers ausmachte. Wie bereits angemerkt wurde, war das besonders nach dem I Weltkrieg die wichtigste Einnahmenquelle für die UGKK. Die polnische Regierung wollte die Frage nach dem Landbesitz und Immobilien systematisieren und demensprechend eine Quote für jede Diözese (Eparchie), Pfarrei und Ordensgemeinschaften erstellen. Der Metropolit Šeptytsky stellte fest, dass die Regierung einer sehr geringen Mindestquote von 3,5 Hektar Land der 1. Klasse für die Pfarrei zugestimmt hat. Anstatt dem vom Staat entnommenen Landbesitz, müsste die Regierung dem Klerus eine Geldleistung zahlen [Hentosh 2016a, 115-28]. Diese Frage war auch deswegen kontrovers, weil für die einen - ostgalizischen Eparchien dies ein Verlust an Landbesitz, für die anderen Lettland, Volynien und Podlachien - die Gebiete, wo die Kirche nur über sehr geringe Immobilien und Landbesitze verfügt hat, großen Gewinn bedeuten könnte. Die Griechisch-Katholische Kirche hat hierbei auf den sozialen Aspekt und Nützlichkeit des Kirchenvermögens aufmerksam gemacht, weil die Kirche damit verschiedene kulturelle, soziale und Bildungsinitiativen unterstützte.

Unabhängig davon hielt es der griechisch-katholische Metropolit für notwendig, die Rechte und Privilegien der Klostergemeinschaften in der Zukunft zu definieren, insbesondere ihre Selbstverwaltung und den Status einer juristischen Person in Finanz- und Vermögensangelegenheiten. In Be- 
zug auf den Landbesitz wurde es als angemessen erachtet, einzelnen Klosterhäusern das Maximum zu erlauben, den gleichen Landbesitz, der die Regierung Privatpersonen erlaubt hat, nämlich 400 bis 600 Hektar zu haben. Die Vorschläge des Metropoliten wurden in den Text des Konkordats im Sinne eines Kompromisses aufgenommen (Art. XXIII) ${ }^{3}$.

\subsection{Bischofswahlen}

Metropolit Šeptytsky hat in einem Brief an den damaligen Nuntius Lorenzo Kardinal Lauri (1864-1941) zusammen mit den anderen hier angemerkten Aspekten deutlich gemacht, dass die Bischöfe frei vom Papst gewählt werden sollen, was auch im Konkordat rezipiert wurde mit der einzigen Änderung, das Präsident der Rzeczpospolita Polska seine Meinung zur Wahl äußern kann ${ }^{4}$.

\subsection{Taufzeugnisse}

Die oben beschriebene Frage nach der Regelung für die Taufzeugnisse sollte neben dem Rituswechsel u.a. auch die Tatsache verdeutlichen, dass die Griechisch-Katholischen Geistlichen keine Staatsbeamten sind, wenn sie auch Dokumente solcher Art erstellen. Dies sollte nach der Ansicht des unierten Episkopats die Einflüsse des Staates auf die kirchlichen Angelegenheiten einstellen und somit das national-ethnische Konfliktpotenzial minimieren. Diese Frage wurde im Konkordat schließlich aufgrund ihrer Partikularität nicht rezipiert.

\subsection{Bildung}

Die unierte Kirche wollte, dass das Konkordat der Katholischen Kirche aller Riten es ermöglicht, katholische Schulen, Universitäten und Bildungseinrichtungen zu gründen. Auch dieser Frage wurde keine große Aufmerksamkeit geschenkt, wobei auch hier positive Schritte erwähnt werden sollen. Dabei geht es um das Artikel XIII des Konkordats, wonach der obligatorische Religionsunterricht in allen öffentlichen Schulen einge-

\footnotetext{
${ }^{3}$ Inter Sanctam Sedem et Poloniae Rempublicam Sollemnis Conventio (2.06.1925), AAS 17 (1925), s. 273-87.

${ }^{4}$ Ibidem.
} 
führt wurde. Zudem konnten die Priester mit ihren wissenschaftlichen Diplomen aus dem Priesterseminar Religionsunterricht in öffentlichen Schulen übernehmen, was eine staatliche Anerkennung des Theologiestudiums bedeutete ${ }^{5}$.

\subsection{Kirchliche Strukturen}

Diese Frage betraf die Struktur der unierten Kirche als solche überhaupt. Nach der Entstehung der II polnischen Republik, wurden die einmal zum Russischen Reich gehörenden Gebiete um Chełm aber auch Lettland, Volynien und Podlachien polnisch. Der Metropolit glaubte, dass die griechisch-katholischen Strukturen, die auf Befehl der russischen Regierung (1875) liquidiert worden waren, nicht kanonisch beseitigt wurden. Die Frage war auch griechisch-katholische Bevölkerung in den Gebieten zu gespitzt, die keine Möglichkeit hatten, ihre geistlichen Bedürfnisse in der Katholischen Kirche byzantinischer Tradition zu erfüllen. Wenn das Konkordat diese Gebiete der lateinischen Kirche unterstellen würde, wäre es „kanonischer Abriss der Diözesen, die solche schrecklichen Ereignisse seitens des zaristischen Russlands überstanden haben“6.

Im Art. IX hat der Apostolische Stuhl nicht nur die Befürchtungen der unierten Kirche erfüllt, wonach die UGKK nun auf Ostgalizien ausgedehnt wurde, sondern auch die Grenzen des polnischen Staates im Sinne von März 1923 anerkannt hat.

\section{REZEPTION DES KONKORDATS AM BEISPIEL DER EPARCHIE STANISLAU}

Am 10. Februar 1925 wurde die Vereinbarung mit der polnischen Regierung unterzeichnet. Als dies aber auch die anderen Aspekte hatten gewisse Auswirkungen auf die unierte Kirche und somit ihre einzelne Strukturen wie Eparchie Stanislau verursacht. Als Ergebnis des Konkordats mussten die griechisch-katholischen Eparchen dem polnischen Staat

\footnotetext{
${ }^{5}$ Ibidem.

6 Tsentralnyi derzhavnyi istorychnyi archiv Ukrainyistorisches Zentralstaatsarchiv der Ukraine u Lvovi. F. 408, op. 1, spr. 51. Zvernennya do Apostol's'koho prestolu, skladene pislya narady v mytropolyta Sheptyts'koho 19 bereznya 1924 r., a. 2-13, 93-94.
} 
die Treue schwören. Die entsprechenden Feierlichkeiten begannen am 8 . September 1925 mit einer festlichen Liturgie in der Warschauer Kathedrale, an der alle katholischen Bischöfe aus Polen teilnahmen.

Nach der Liturgie zogen die Bischöfe in das Belvedere Palais. In der Residenz des Präsidenten schworen alle katholischen Bischöfe ihrerseits in Anwesenheit vor Ministerpräsidenten, Minister für religiöse und öffentliche Bildung, und dem päpstlichen Nuntius persönlich einen Eid dem polnischen Präsidenten gegenüber. Der feierliche Eid jedes Bischofs, einschließlich der ukrainischen, hatte folgende Bedeutung: „Ich schwöre bei Gott und dem heiligen Evangelium, und ich verspreche, wie es dem Bischof gehört, der Treue der Rzeczpospolita Polska. Ich schwöre und verspreche, dass ich die verfassungsmäßige Regierung mit voller Loyalität respektiere und dies tun werde, damit meine Geistlichkeit ihn ehren wird. Ich verspreche auch, dass ich nicht an Sitzungen teilnehmen und keine Vereinbarung treffen werde, die dem polnischen Staat schaden oder die öffentliche Ordnung verletzen könnte. Ich werde meinen Geistlichen nicht erlauben, an solchen Veranstaltungen teilzunehmen. Kümmernd sich um die Güte und die Interessen des Staates werde ich versuchen, ihn vor jeder Gefahr zu bewahren, welche Bedrohung für mich bekannt wird“ [Pylypiv 2013, 153-62].

In einem Artikel des Konkordats kommt folgendes zur Sprache: „Die katholische Kirche wird ohne Rücksicht auf den Ritusunterschied volle Freiheit im polnischen Staat genießen. Der Staat versichert der Kirche die freie Ausübung ihrer Macht und Jurisdiktion, ebenso die freie Verwaltung und Leitung ihrer Angelegenheiten und ihrer Vermögen gemäß den Gesetzen Gottes und dem kanonischen Recht (Art. I)“.

Das Konkordat bestätigte das Bestehen der Kirchenprovinz Lviv des griechisch-russischen (ruthenischen) Ritus mit der Erzeparchie Lviv und den Eparchien Peremyšl und Stanislau (Art. IX). Somit entfall auch die Bitte der unierten Bischöfe, die griechisch-katholischen Gläubigen außerhalb des Ostgaliziens der lateinischen Kirche zu unterstellen.

Gemäß diesem Dokument fiel die Wahl von Erzbischöfen und Bischöfen in die Kompetenz des Heiligen Stuhls. Seine Heiligkeit sei damit einverstanden, dass man sich vor der Ernennung von Erzbischöfen, Bischöfen der Erzeparchie, Koadjutoren mit dem Recht der Nachfolge sowie auch eines Militärbischofs schriftlich an den Präsidenten der Rzeczpospolita (Republik) wendet, um sich zu vergewissern, dass er im politischen Sinne 
Die Auswirkungen des Konkordats zwischen dem Apostolischen Stuhl 241

gegen diese Wahl nichts einzuwenden hätte. Die gewählten Bischöfe wurden verpflichtet, dem Präsidenten der Republik einen Treueid zu leisten (Art. XI).

Das Konkordat sah vor, wie bereits angemerkt, dass an allen öffentlichen Schulen außer Hochschulen das Fach Religion obligatorischen Charakter habe. Dieses Fach sollen für katholische Schüler die von den Schulräten ernannten Lehrer unterrichten, die ausschließlich aus den von den Ordinarien für den Religionsunterricht bevollmächtigten Personen gewählt werden. Die zuständige kirchliche Autorität hatte bezüglich des Religionsunterrichts, auf dessen Inhalt und auf die moralische Lebensführung der Lehrer Acht zu geben. Falls einem Lehrer die vom Bischof früher erteilte Bevollmächtigung entzogen worden war, durfte dieser nicht mehr im Fach Religion unterrichten. Dieselben Prinzipien, die sich auf die Wahl und Entlassung der Schullehrer bezogen, bezogen sich auch auf die Professoren und Dozenten der katholischen Fakultäten an den staatlichen Universitäten (Art. XIII).

In jeder Diözese oder Eparchie hatte die katholische Kirche die Möglichkeit, ein Priesterseminar gemäß dem Kirchenrecht zu haben. Dies bestätigte die Existenz und Funktion des Stanislauer Priesterseminars, das 1907 gegründet worden ist. Die Bitte über die Gründung von katholischen Schulen und Universitäten wurde im Konkordat nicht rezipiert. Die Ernennung der Lehrer für ein Priesterseminar gehörte ausschließlich in die Kompetenz der kirchlichen Autorität. Die von den „großen“ Priesterseminaren (theologischen Hochschulen) erteilten Diplome waren gültig für den Religionsunterricht in allen öffentlichen Schulen, außer Hochschulen. Laut Art. XIV wurden Güter, die der Kirche gehörten, keinem juristischen Akt unterstellt. Ohne Zustimmung der kirchlichen Autorität war es nicht möglich, deren Bestimmung zu ändern.

In jeder Diözese oder Eparchie sollte eine vom Bischof nominierte (benannte) Kommission im Einverständnis mit dem zuständigen Minister gebildet werden, die die Aufgabe hatte, in den Kirchen und in den der Kirche angehörenden Räumen Antiquitäten, Kunstwerke, Archivalien und Handschriften, die einen historischen und künstlerischen Wert hatten, aufzubewahren. Von Art. XV des Konkordats wurde vorgesehen, dass die Geistlichen, deren Vermögen sowie die Güter der kirchlichen und klösterlichen Rechtspersonen (juristische Personen) einer Besteuerung unterliegen, genauso wie die Personen und das Vermögen der anderen Bürger der 
Republik und weltlichen juristischen Personen der Republik. Eine Ausnahme wurde nur für die Gottesdienste bestimmten Häuser, Priesterseminare, Häuser für die Ausbildung von Ordensbrüdern und Schwestern, Wohnhäuser für Ordensleute, die das Armutsgelübde abgelegt haben, gemacht (Art. XV).

Die Rzeczpospolita garantierte für den Klerus folgende Dotationen: für Kardinäle - 800 Złoty, für Erzbischöfe - 600 Złoty, für Diözesanbischöfe 600 Złoty (Art. XXIV). Dotationen wurden garantiert auch für Weihbischöfe, Mitglieder der Domkapitel, Pfarrer, Rektoren der Filialkirchen, Kapläne und Mitarbeiter des Konsistoriums, Professoren und Priesteramtskandidaten, Lehrer der theologischen Institute. Ferner wurden Dotationen für die Rente der Ruheständler, den Unterhalt der Kathedralchöre und die niederen Kirchendiener garantiert. Der Staat deckte auch Kosten für die Pastoralvisitationen der Bischöfe, den Unterhalt der Konsistorien, die Führung von Pfarreibüchern und Postgebühren. Es wurden auch ein Jahresbaufonds und eine jährliche Unterstützung für Kirchen und kirchliche Institutionen gebildet.

Doch, wie die Archivakten zeugen, hielten sich die meisten Priester der Eparchie Stanislau nicht an die Forderungen der Vereinbarung. Zum Bespiel mussten die Priester während des Gottesdienstes nach Art. VIII Gebete für die Republik Polen und den polnischen Präsidenten abhalten. Aber wie der Meldung des Wojewoden von Stanislau an das Ministerium für Glaubensangelegenheiten und Bildung vom 11. Januar $1927 \mathrm{zu}$ entnehmen ist, machten das fast $90 \%$ griechisch-katholische Geistlichen nicht [Jehreshij 2003, 94-95]. Solidarisch mit ihren Priestern waren auch die Gläubigen. Das bewirkte das Misstrauen der politischen Macht Bischof Hryhorij gegenüber. So beauftragte das Ministerium für Glaubensangelegenheiten und Bildung seine Beamten in einer entsprechenden Behörde in Stanislau, die Hirtenbriefe des Eparchialbischofs, Exemplare der Zeitschrift „Visnyk Stanyslaviwskoï Jeparchiï“ (Bulletin der Eparchie Stanislau), Berichte über die Tätigkeit von religiösen Organisationen, statistische Angaben und andere Informationsmaterialien zu sammeln und dem Ministerium zukommen zu lassen [ibidem, 90].

Es sei bemerkt, dass Kyr Hryhorij eine große Bedeutung für die Gestaltung der kirchlich-staatlichen Beziehungen in der Eparchie Stanislau in der Zwischenkriegszeit hatte und der Beschluss des Konkordats von 1925 zu implementieren ersuchte. Als regierender Erzhirte der Eparchie Sta- 
nislau gab eben er den Ton in diesen Beziehungen an. Auf seine Entwicklung hatte das Studium in Wien einen wesentlichen Einfluss. Dort hatte der künftige Bischof die spezifischen Tätigkeitsfelder der römisch-katholischen Priester näher kennengelernt und die gesamte Orientierung der westlichen Kirche studiert. Meiner Meinung nach, bemühte sich Bischof Hryhorij das Wohl der Kirche und des ukrainischen Volkes zu fördern. Deswegen wollte er die Beziehungen zwischen der Kirche und dem Staat sowie die Zusammenarbeit mit der Regierung nicht zuletzt mithilfe des Konkordats von 1925 verbessern, normalisieren und rief immer zum Frieden und zur Ausbreitung des Reiches Gottes auf.

\section{RESÜMEE}

Nach dem Zusammenbruch der Mittelmächte strebten sowohl das ukrainische, als auch das polnische Volk die Wiederherstellung eines eigenen Staates an. Hierzu benötigte es Unterstützung, die die Nation unter anderem in der katholischen Kirche und dem Papst fand. Nach dem Konkordat mit dem Heiligen Stuhl 1925 ging es mit den kirchlichen Organisationen steil bergauf: Trotz leichten Problemen mit der republikanischen Regierung stieg die Zahl der Priester um 40\% auf beinahe 13000 an. Die Zahl sowie die Tätigkeit staatlich geförderter kirchlicher Vereine und Verbände nahmen erheblich zu. Der polnische Staat präsentierte sich bis zum Zweiten Weltkrieg als katholischer Staat. „Die katholische Religion durchdrang und festigte damals durch ihre nationale Grundhaltung den gesamten polnischen Staat mit" .

Der ukrainische Rechtswissenschaftler Stepan Baran charakterisierte die Rolle des Konkordats von 1925 als sehr bedeutend. Seiner Ansicht nach hat die Ukrainische Griechisch-Katholische Kirche dieser Vereinbarung zwischen dem polnischen Staat und Apostolischen Stuhl viel zu verdanken, wenn auch ihr ein Teil ihres Immobilienvermögens mit dem zusätzlichen Vertrag vom 20. Juli $1938^{8}$ entnommen wurde. Hätte es nicht

\footnotetext{
${ }^{7}$ Vgl. Die Rolle der katholischen Kirche in der polnischen Geschichte seit 1795, http:// www.puhli.de/uni/papers/kirche-in-polen/geschichte.html [eingesehen am: 17.04.2019].

8 Am 20. Juni 1938 wurde ein zusätzlicher Vertrag zum Konkordat von 1925 zwischen Polen und dem Vatikan unterzeichnet. Nach der neuen Vereinbarung hat der Vatikan von der polnische Regierung 2,5 Millionen złoty ausgezahlt bekommen, wo-
} 
das Konkordat gegeben, wäre die Lage sowie die Position der Katholiken des byzantinischen Ritus genauso beträchtlich, wie die der vielen ukrainischen (ruthenischen) Bürger [Baran 1940], die unter polnischer Regierung gelitten haben. An der anderen Stelle verdeutlichte er, dass das Konkordat die UGKK beschützt und ihr eine breitere Rechtsweite im Vergleich zum österreichischen Gesetz vom 7. Mai 1874 gegeben hat [Baran 1947]. Unumstritten ist, dass die Klärung der Rechtsverhältnisse zur gewissen Stabilität in der Verwaltungs- und Seelsorgestrukturen der UGKK und der Eparchie Stanislau geführt, deren Leitung sich dem Staat gegenüber weiterhin loyal präsentierte. Nichtsdestotrotz spielte in der Zwischenkriegszeit nunmehr nicht unbedingt die unierte Kirche, sondern die ukrainische Elite mit ihren oft radikalen und volksorientierten Parteiungen eine einflussreichere Rolle in den national-ethnischen Prozessen, was die Rezeption und Auswirkungen des bedeutenden Konkordats von $1925 \mathrm{zu}$ erschweren vermochte.

\section{LITERATURVERZEICHNIS}

Baran, Stepan. 1940. "Tserkovne pytannia w Polshchi." https://zbruc.eu/node/353 74 [eingesehen am: 16.04.2019].

Baran, Stepan. 1947. Mytropolyt Andrei Sheptyts'kyi. Zhyttia i dijalnist. München: Vernyhora.

"Die Rolle der katholischen Kirche in der polnischen Geschichte seit 1795." http:// www.puhli.de/uni/papers/kirche-in-polen/geschichte.html [eingesehen am: 17.04.2019].

Hentosh, Liliana. 2016a. "Rol'metropolyta Andreia Sheptyts'koho v ukladenni konkordatu mizh Apostolskym Prestolom i Polshcheju (1925).” Posen 10:115-28.

Hentosh, Liliana. 2016b. "Hreko-katolytskoji tserkvy u Polshchi do pidpysannja konkordatu 1925 r." Eminak 1(3): 21-25.

Jehreshij, Oleh. 2003. Suspilno-politychna ta kulturalno-prosvitnycka dijalnist jepyskopa Hryhorija Chomyšyna (1904-1945 rr). Ivano-Frankivsk: Prykarpats'kyy natsional'nyy universytet imeni Vasylya Stefanyka.

Mroěz, Maciej. 2004. W kregu dyplomacji watykaněskiej. Rosja, Polska, Ukraina w dyplomacji Watykaněskiej w latach 1917-1926. Toruně: Wydawnictwo Adam Marszałek.

nach der Apostolische Stuhl sich auf seine Rechte an Kirchenvermögen, Landbesitz, Kirchen und Kapellen in den Gebieten, die jemals zum russischen und preußischen Reichen gehörten, verzichtete. Demzufolge hat die unierte Kirche ihren dortigen Besitz verloren [Pylypiv 2013, 153-62]. 
Perewesij, Vitaliy. 1998. Hreko-katolytska tserkvy v umovach ukrainsko-polskoi konfrontacii 20 roky $X X$ st. Kiev: Natsional'na akademiya nauk Ukrayiny, Instytut politychnykh ta etnonatsional'nykh doslidzhen'.

Pylypiv, Ihor. 2013. "Pravovyj status hreko-katolytskoi tserkvy d druhij Rechi Pospolytij (1925-1939)." Efektyvnist derzhavnoho upravlinnia (Effektivität der staatlichen Verwaltung) 34:153-62.

Wolf, Hubert. 2010. Pope and Devil. The Vatican's Archives and the Third Reich. Cambridge: The Belknap Press of Harvard University Press.

\section{Die Auswirkungen des Konkordats zwischen dem Apostolischen Stuhl und Polen von 1925 auf die Eparchie Stanislaviv}

\section{Zus a m m enfassung}

Nach dem Zusammenbruch der Mittelmächte versuchten das ukrainische und polnische Völker, ihre eigenen Staaten wiederherzustellen. Diese Bemühungen wurden wesentlich durch die ethnopolitische Situation in Galizien erschwert, insbesondere nach der Entscheidung der Entente vom 15. März 1923, Ostgalizien an Polen anzuschließen. Für die ukrainische griechisch-katholische Kirche (UGKK) ergab sich eine weitere Chance und Herausforderung - mit einer neuen Regierung und Wirklichkeit zusammenzuarbeiten. Der geopolitische Wandel verursachte eine Art von rechtlichem Nihilismus in Bezug auf das Staatskirchenrecht, was sich als selektive Gerechtigkeit herausstellte. Diese ungeklärten Rechtsverhältnisse sollten das Konkordat zwischen dem Apostolischen Stuhl und dem polnischen Staat aufheben. Die Hauptvoraussetzungen für die Ausarbeitung des Konkordats seitens der ukrainischen griechisch-katholischen Kirche waren Rechtsunsicherheit, finanzielle und wirtschaftliche Schwierigkeiten sowie gesellschaftspolitische Fragen in der Gesellschaft. Die Kirche des byzantinischen Ritus wurde von Metropolit Šeptytsky im Auftrag seiner Bischöfe von Stanislau (Stanislaviv) und Peremyšl und deren Gläubigen in diesen Vorbereitungsprozess eingeführt. Für die UGKK war es wichtig gewesen, die Fragen des kirchlichen Eigentums, der Bischofswahlen, der Taufscheine, des Bildungswesens und der kirchlichen Strukturen zu klären. Das am 10. Februar 1925 unterzeichnete Abkommen war gegenüber allen Bischöfen des UGKK, einschließlich Bischof Hryhori Chomyšyn (1867-1945) verbindlich und setzte für sie die Verpflichtung zur Loyalität dem polnischen Präsidenten und Rzeczpospolita Polska gegenüber voraus.

Schlüsselwörter: Konkordat; Eparchie Stanislau (Stanislaviv); GriechischKatholische Kirche; Polen-Ukraine 


\title{
The Impact of the 1925 Concordat between the Apostolic See and the Republic of Poland on the Eparchy of Stanislaviv
}

\begin{abstract}
Su m m a r y
After the collapse of the Central Powers, the Ukrainian and Polish authorities tried to restore their own states. These attempts were essentially hindered by the ethnopolitical situation in Galicia, especially after the decision of the Entente on 15 March 1923 to append East Galicia to Poland. The Ukrainian Greek-Catholic Church (UGCC) faced another opportunity and challenge - to cooperate with a new government under different circumstances. Such a geopolitical change led to a kind of legal nihilism concerning Church-State relations, which introduced selective justice. These legal relations should have been resolved by way of a ruling (concordat) between the Apostolic See and the Polish State. The chief preconditions for the drafting of a concordat by the Ukrainian Greek-Catholic Church were: legal uncertainty, financial and economic difficulties, and the sociopolitical issues in society. In this preparatory process, the Byzantine rite Church was led by Metropolitan Šeptytsky, who spoke on behalf of his bishops of Stanislaviv and Przemyśl and the faithful. It was important for the UGCC to clarify the questions of ecclesiastical property, elections of bishops, baptismal certificate, education, and ecclesiastical structures. The agreement, signed on February 10th, 1925, obliged all UGCC bishops to take an oath of allegiance, including Bishop Hryhorij Chomyšyn (1867-1945), to the Polish President and pledge their loyalty to the state.
\end{abstract}

Key words: Concordat; Eparchy of Stanislaviv; Greek Catholic Church; PolandUkraine

\section{Wpływ konkordatu między \\ Stolicą Apostolską a Rzecząpospolitą Polską z 1925 r. na Eparchię Stanisławowską}

\section{Streszczenie}

Po upadku państw centralnych władze ukraińska i polska próbowały przywrócić własne państwo. Próby te były zasadniczo komplikowane przez sytuację etniczno-polityczną w Galicji, zwłaszcza po decyzji Ententy z 15 marca 1923 r. o połączeniu Galicji Wschodniej z Polską. Dla ukraińskiego Kościoła greckokatolickiego (UKGK) rozpoczęła się kolejna szansa i wyzwanie - współpraca z nowym rządem i rzeczywistością. Taka zmiana geopolityczna spowodowała pewnego rodzaju nihilizm prawny $\mathrm{w}$ stosunku do relacji państwa i kościoła, które okazało się selektywną sprawiedliwością. Te stosunki prawne powinien był rozstrzygać konkordat między Stolicą Apostolską a państwem polskim. Głównymi warunkami wstępnymi przygotowania konkordatu ze strony ukraińskiego Kościoła greckokatolickiego były niepewność prawna, napięte trudności finansowe i gospodarcze oraz kwestie społeczno-polityczne w społeczeństwie. Kościół obrządku bizantyjskiego $\mathrm{w}$ tym procesie przygotowawczym był prowadzony przez metropolitę Szeptyckiego, który przemawiał w imieniu swoich biskupów ze Stani- 
sławowa i Przemyśla oraz ich wiernych. Ważne dla UKGK było wyjaśnienie kwestii własności kościelnej, wyborów biskupów, świadectwa chrztu, edukacji i struktur kościelnych. Umowa, podpisana 10 lutego 1925 r., zobowiązała do złożenia przysięgi wierności wszystkich biskupów UKGK, w tym biskupa Grzegorza Chomyszyna (18671945), prezydentowi polskiemu i zobowiązała do lojalności wobec państwa.

Słowa kluczowe: konkordat; Eparchia Stanisławów; Kościół greckokatolicki; PolskaUkraina

Informacje o Autorze: Ks. MGR LIC. MYKHAILO KLAPKIV - doktorant, Katedra Prawa Katolickich Kościołów Wschodnich, Instytut Prawa Kanonicznego, Wydział Prawa, Prawa Kanonicznego i Administracji Katolickiego Uniwersytetu Lubelskiego Jana Pawła II; adres do korespondencji: Al. Racławickie 14, 20-950 Lublin, Polska; e-mail: mykhailo.klapkiv@ukr.net; https://orcid.org/0000-0001-5276-5265 